\title{
Global tobacco consumption: what does it mean for urologists?
}

\author{
Alexandra M Hay
}

World No Tobacco Day 2006, organized by the WHO's Tobacco Free Initiative, passed by on the 31st May. Although the damaging effects of smoking are well known, tobacco consumption remains the "single largest preventable cause of cancer in the world today" (www.who.int/tobacco). The Tobacco Free Initiative reminds us that smoking is not only associated with many serious and chronic diseases, but also exacerbates poverty, a fact that is often forgotten. The increased burden on healthcare and the direct costs of smoking have the greatest impact on the poorest individuals, and yet it is often those people who are more likely to take up smoking.

With much of the available information focusing on smoking-related respiratory disease, the urologic implications of smoking are often overlooked. A recent antismoking campaign in the UK highlighted a number of less well publicized smoking-related diseases and conditions, including erectile dysfunction.

The June issue of Nature Clinical Practice Urology includes a review on the epidemiology of bladder cancer. The authors highlight the fact that much is known about the environmental risk factors associated with developing bladder cancer, including tobacco smoking and occupational exposure to carcinogens. They discuss the trends for the decrease in bladder cancer-related mortality in many developed countries, citing data from Japan, the US, and some European countries, as a result of reduced exposure to environmental risk factors. With the introduction of smoking bans in some public places in countries such as Ireland, Scotland and New Zealand, along with hard-hitting antismoking campaigns, many countries are observing a reduced uptake of smoking and an increase in numbers of people quitting. This trend is in direct contrast to what is happening in many other less developed countries, and there seem to be two quite different patterns of tobacco exposure emerging. Pelucchi et al. use the example of

\section{There have \\ been calls \\ for medical \\ professionals \\ to lead by \\ example, and \\ to...influence \\ patient \\ behavior by \\ promoting \\ smoking \\ cessation.}

AM Hay is Editor of Nature Clinical Practice Urology

\section{Competing interests}

The author declared she has no competing interests

www.nature.com/clinicalpractice doi:10.1038/ncpuro0513 certain countries in Eastern Europe with trends towards increased bladder cancer mortality without the declines in tobacco consumption seen in many other European countries.

China is not only the most populous country in the world, it also has the highest consumption of tobacco; with over 320 million smokers, it accounts for about one-third of all smokers worldwide (Respirology 8: 17-21). Despite the efforts of the Chinese government to halt the rise in smoking by restricting tobacco advertising and sales, and by promoting abstinence from smoking, Chinese teenagers continue to take up smoking in increasing numbers (an estimated 9 million Chinese teenagers are smokers). This has huge implications for the health of the Chinese population, with deaths from smoking-related causes currently over 1 million per year, and estimated to rise to 3 million per year by 2050, if the current patterns of smoking continue.

There have been calls for medical professionals to lead by example, and to use their unique position to influence patient behavior by promoting smoking cessation. The fact that medical students are more likely to take up smoking when they start their medical training than to give it up suggests that many education programs have little impact on their behavior (Thorax 54: 70-78). The Smokescreen Education Program, developed by a team at the University of Sydney, is targeted at educating medical students about the effects of smoking and how they can help their patients to give up. This program has been translated into several languages and tested in many countries, including China.

With the continued efforts of antismoking campaigns and education programs, the huge predicted death-toll in countries such as China might be avoided. In the future, it is hoped that more countries will experience trends of reduced incidence in mortality from bladder cancer, such as those highlighted by Pelucchi and colleagues. 